\title{
A PROSPECTIVE STUDY ON THE GASTROGRAFIN IN THE MANAGEMENT OF ADHESIVE SMALL BOWEL OBSTRUCTION
}

\author{
Nitin Nangare1, Basavaraj Nagur², Sangeeta Biradar 3 , Mahesh Reddy S $^{4}$, Jignesh Kumar Savsaviya ${ }^{5}$, Madhavendra Kabra 6 , \\ Shruti Panicker ${ }^{7}$, Aditya Phadhe ${ }^{8}$
}

\begin{abstract}
${ }^{1}$ Assistant Professor, Department of Surgery, Krishna Institute of Medical Sciences, Karad.
${ }^{2}$ Resident, Department of Surgery, Krishna Institute of Medical Sciences, Karad.

${ }^{3}$ Resident, Department of Surgery, Krishna Institute of Medical Sciences, Karad.

${ }^{4}$ Resident, Department of Surgery, Krishna Institute of Medical Sciences, Karad.

${ }^{5}$ Resident, Department of Surgery, Krishna Institute of Medical Sciences, Karad.

${ }^{6}$ Resident, Department of Surgery, Krishna Institute of Medical Sciences, Karad.

${ }^{7}$ Resident, Department of Surgery, Krishna Institute of Medical Sciences, Karad.

${ }^{8}$ Resident, Department of Surgery, Krishna Institute of Medical Sciences, Karad.
\end{abstract}

\section{ABSTRACT}

\section{OBJECTIVE}

The purpose of this study was to determine the diagnostic and therapeutic role of hyperosmolar water-soluble contrast medium gastrografin in cases of small bowel obstruction.

\section{BACKGROUND}

Adhesive small bowel obstruction is a common emergency problem; post-operative abdominal adhesions represent the main aetiological factor for intestinal obstruction. It is commonly used for the diagnosis of small bowel obstruction, but it also has a therapeutic role in Small Bowel Obstruction (SBO).

\section{METHODS}

A prospective Gastrografin study was performed in patients with small-intestine obstruction from June 2013 to June 2015 in Krishna Hospital, Karad. Patients with clinical evidence of adhesive small bowel obstruction were given trial conservative treatment, unless there was suspicion of strangulation. Those who responded in the initial 48 hours had conservative treatment continued. Patients showing no clinical and radiologic improvement in the initial 48 hours were randomized to undergo either Gastrografin meal and follow-through study or surgery. Contrast that appeared in the large bowel within 24 hours was regarded as a partial obstruction and conservative treatment was continued. Patients in whom contrast failed to reach the large bowel within 24 hours were considered to have complete obstruction and laparotomy was performed. For patients who had conservative treatment for more than 48 hours with or without Gastrografin, surgery was performed when there was no continuing improvement.

\section{RESULTS}

The 100 patients were subjected to conservative management for $48 \mathrm{~h}$ under close observation. Within 48 hours 10 patients with SBO obstruction resolved, another 4 cases developed signs of peritoneal irritation and were explored. The other 86 cases that did not resolve conservatively within $48 \mathrm{~h}$ were subjected to gastrografin administration through a nasogastric tube. Among these, $69(80.2 \%)$ patients' symptoms has been resolved by gastrografin, and 17 (19.7\%) patients undergone surgery.

\section{CONCLUSION}

Gastrografin can be used in the case of SBO; it is safe and reduces the need for surgery and hospital stay and morbidity when conservative treatment fails.

\section{KEYWORDS}

Gastrografin, Small Bowel Obstruction, Surgery.

HOW TO CITE THIS ARTICLE: Nangare N, Nagur B, Biradar S, et al. A prospective study on the gastrografin in the management of adhesive small bowel obstruction. J. Evolution Med. Dent. Sci. 2016;5(46):2889-2891, DOI: 10.14260/jemds/2016/673

\section{INTRODUCTION}

Intestinal obstruction is a partial or complete blockage to the passage of intestinal contents through the gastrointestinal

Financial or Other, Competing Interest: None.

Submission 18-03-2016, Peer Review 14-04-2016,

Acceptance 20-04-2016, Published 08-06-2016.

Corresponding Author:

Dr. Basavaraj Nagur,

Room No. 20,

IHR Hostel,

KIMS University,

Karad.

E-mail: sachin.nagur@gmail.com

DOI: $10.14260 /$ jemds $/ 2016 / 673$ tract and is a major indication for urgent abdominal surgery. More than two-thirds of all small bowel obstructions were due to peritoneal adhesions. ${ }^{[1]}$ followed by neoplasms and hernias. The exact cause of adhesive SBO is not known. Gynaecological surgery and appendectomy are the most common cause of SBO.[2] These patients are usually difficult to diagnose and manage. Patients with strangulation require emergency operation, but management of remaining patients is more difficult.[3]

Adhesive obstruction may occur at any time after surgery, about $20 \%$ of the obstructions appeared more than 10 years after the initial abdominal operation.[4]

Gastrografin, a water soluble contrast medium, has been found useful in the management of adhesive small bowel obstruction. A few studies reported that gastrografin could 
accurately predict the need for surgical treatment.[5,6] makes the fluid move into the intestinal lumen and increases pressure gradiant across obstructive sites that may result in resolving the obstruction. ${ }^{[7,8,9]}$

The present prospective study was designed to investigate the value of the hyperosmolar water-soluble contrast medium Gastrografin $₫$ in choosing when conservative treatment might be appropriate for patients with small-intestine obstruction caused by post-operative adhesions.

\section{MATERIALS AND METHODS}

A prospective Gastrografin study was performed in patients with small-intestine obstruction from June 2014 to June 2015 in Krishna Hospital, Karad; 100 patients were included in this study as per category.

\section{Inclusive Category}

- All the Patients admitted through the emergency room to the Department of Surgery, Krishna Institute of Medical Science, Karad.

- Patients with clinical and radiologic evidence of adhesive small bowel obstruction were included.

- Exclusive category.

- Patients with signs of strangulation excluded.

- Patients with documented intra-abdominal malignancy, inflammatory bowel disease or history of abdominal irradiation were excluded.

A detailed history including information on previous abdominal surgery and adhesive obstruction was taken and a complete physical examination was performed for every patient. A nasogastric tube was inserted for decompression with strict measurement of output. Intravenous fluid replacement was given and electrolyte imbalances were corrected as required. Supine and erect abdominal radiographs were taken. Patients with clinical evidence of adhesive small bowel obstruction were given trial conservative treatment.

Patients with obstruction that improved clinically or radiologically in the initial $48 \mathrm{~h}$ continued to receive conservative treatment. Clinical improvement was defined as the presence of decreased abdominal pain, distension, tenderness or nasogastric tube output or bowel opening if the patient had constipation on admission. Radiological improvement was defined as a decrease in number of dilated bowel loops or in the diameter of dilated small bowel. Those who responded in the initial 48 hours had conservative treatment continued.

Patients showing no clinical and radiologic improvement in the initial 48 hours were randomized to undergo either Gastrografin meal and follow-through study or surgery. Gastrografin $(40 \mathrm{~mL}$ ) was mixed with $40 \mathrm{~mL}$ of distilled water and administered through a nasogastric tube, which was unclamped after 1 hour. Direct abdominal radiographs were obtained after 4 and 8 hours.

Contrast that appeared in the large bowel within 8 hours was regarded as a partial obstruction and conservative treatment was continued. Patients in whom contrast failed to reach the large bowel within 8 hours and patient with fever, persistent abdominal pain or signs of peritonitis were considered to have complete obstruction and laparotomy was performed. For patients who had conservative treatment for more than 48 hours with or without Gastrografin, surgery was performed when there was no continuing improvement.

\section{RESULTS}

100 patients were admitted for attacks of SBO. Their age ranged from 20-70 years. Out of these 100 patients, 90 patients presented with complaints of pain abdomen and 81 patients with constipation and 38 with vomiting, thus making most common symptom as pain abdomen (Shown in Table No. 1). There were 68 male patients and 32 females (Shown in Fig. No. 1).

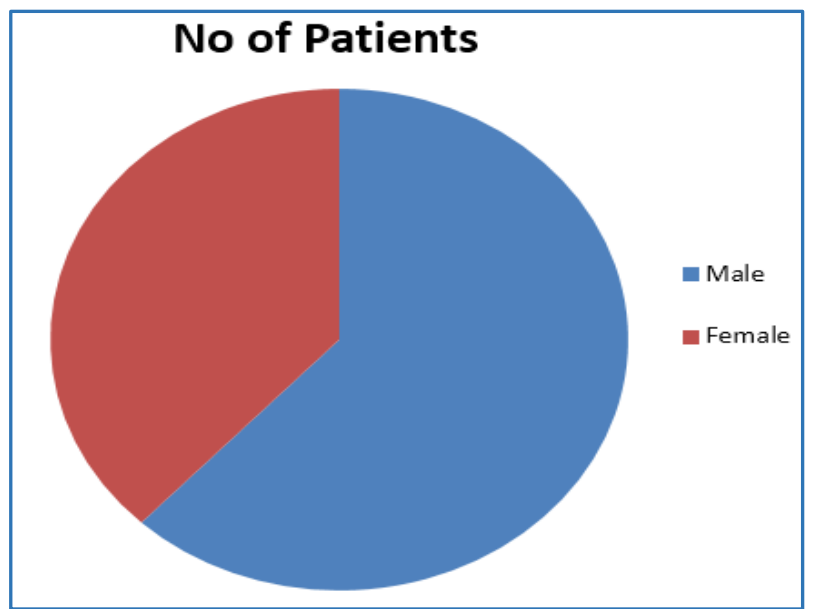

Fig. 1: Shows Number of Patients with Sex Ratio

\begin{tabular}{|c|c|}
\hline $\begin{array}{l}\text { Presenting } \\
\text { Symptoms }\end{array}$ & $\begin{array}{l}\text { Percentage of } \\
\text { Patients }\end{array}$ \\
\hline Abdominal Pain & $90 \%$ \\
\hline Constipation & $81 \%$ \\
\hline Vomiting & $38 \%$ \\
\hline
\end{tabular}

The 100 patients were subjected to conservative management for $48 \mathrm{~h}$ under close observation. Within 48 hours 10 patients with SBO obstruction resolved, another 4 cases developed signs of peritoneal irritation and were explored. The other 86 cases that did not resolve conservatively within $48 \mathrm{~h}$ were subjected to gastrografin administration through a nasogastric tube. Among 86 patients, $39(45.34 \%)$ patient's gastrografin seen in the caecum within 4 hours and $21(24.41 \%)$ patient's gastrografin seen in the caecum within 8 hours, $9(10.46 \%)$ patient's gastrografin after 8 hours, 17 (19.76\%) patients underwent surgery (Shown in Fig. 2). Among the 17 patients who has undergone surgery, 11 patients had the history of undergoing abdominal surgeries more than once in the past for various reasons. 


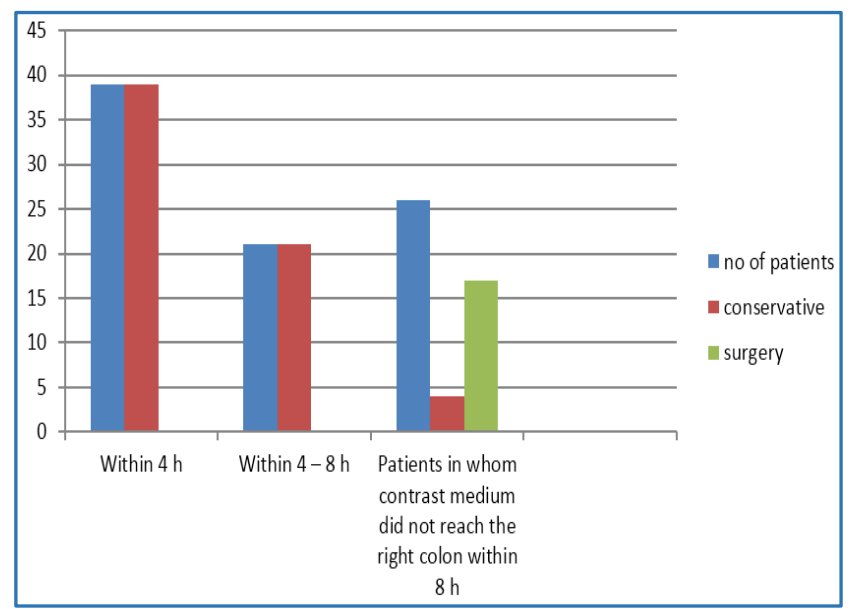

Fig. 2: Shows Number of Patients and their Management

\section{DISCUSSION}

Adhesive small bowel obstruction is a common surgical problem. Any abdominal surgery in past can lead to adhesive SBO and incidence of complication varies from $6 \%$ to $30 \%$.[10] Appendicectomy and colorectal surgery are the common surgical procedures, which can lead to adhesive small bowel obstruction in different studies.[11]

Yet, there has been no standard protocol for its management. In the absence of bowel strangulation, adhesive obstruction can be treated conservatively. However, the optimal duration of this trial conservative treatment is controversial.

Gastrografin is the contrast medium most commonly used. It is an ionic bitter-flavoured mixture of sodium diatrizoate, meglumine diatrizoate, and a wetting agent (Polysorbate 80). The osmolarity is $1900 \mathrm{mOsm} / \mathrm{L}$, approximately six times that of extracellular fluid. It promotes shifting of fluid into the bowel lumen and increases the pressure gradient across an obstructive site.

The bowel content is diluted, and in the presence of the wetting agent passage of bowel contents through a narrowed lumen is facilitated. Gastrografin also decreases oedema of the bowel wall and enhances bowel motility. Barium has also been used to evaluate adhesive small bowel obstruction; it is not as easily diluted by enteric fluid as Gastrografin and provides a better mucosal image on radiography. However, a barium study can be risky, because it may become inspissated and completely obstruct the bowel. Barium may spread into the peritoneal cavity if perforation occurs, a condition that is potentially lethal. Gastrografin is water-soluble and relatively safe even if the obstruction is complicated by perforation. Complications from the use of Gastrografin in small bowel obstruction are rare.

The risk factors associated with failure of conservative treatment remain poorly understood. The importance of nasogastric tube output and size of dilated small bowel have seldom been evaluated in the literature. We found that nasogastric tube output was significantly greater in patients who failed to respond to conservative treatment versus those successfully treated with conservative treatment. An alternative explanation is that the nasogastric tube drainage of patients who responded to conservative treatment decreased with time.

In this study concluded that gastrografin administration in SBO is safe; it can be used therapeutically because it has a role in resolution of SBO. Gastrografin can also help in early diagnosis of cases of SBO who requires surgery. Thus, it also shortens the hospital stay and morbidity.

\section{REFERENCES}

1. Menzies D. Postoperative adhesions: their treatment and relevance in clinical practice. Ann R Coll Surg Engl 1993;75(3):147-53.

2. Abbas S, Bisset IP, Parry BR. Meta-analysis of oral water soluble contrast agent in the management of adhesive small bowel obstruction. Br J Surg 2007;94(4):404-11.

3. Choi HK, Law WL, Chu KW. Therapeutic value of gastrografin in adhesive bowel obstruction after unsuccessful conservative treatment: a prospective randomized trial. Ann Surg 2002;236(1):1-6.

4. Menzies D, Ellis $H$. Intestinal obstruction from adhesions- how big is the problem? Ann R Coll Surg Engl 1990;72(1):60-3.

5. Onoue $\mathrm{S}$, Katoh $\mathrm{T}$, Shibata $\mathrm{Y}$, et al. The value of contrast radiology for postoperative adhesive small bowel obstruction. 2002;49(48):1576-8.

Hepatogastroenterology

6. Chen SC, Lin FY, Lee PH, et al. Watersoluble contrast study predicts the need for early surgery in adhesive small bowel obstruction. Br J Surg 1998;85(12):1692-4.

7. Burge J, Abbas SM, Roadley G, et al. Randomized controlled trial of gastrografin in adhesive small bowel obstruction. ANZ J surg 2005;75(8):672-4.

8. Gorecki W, Krysta M, Bysiek A, et al. Gastrografin challenge test for the management of subileus in children. Przegl Lek 2007;64(3):53-5.

9. Farid M, Fikry A, Nakeeb AE, et al. Clinical impacts of gastrografin follow-through in adhesive small bowel obstruction. J Surg Res 2010;162(2):170-6.

10. Assalia A, Schein M, Kopelman D, et al. Therapeutic effect of oral gastrografin in adhesive, partial small-bowel obstruction: a prospective randomized trial. Surgery 1994;115(4):433-7.

11. Strodahl A, Laerum F, Gjolberg T, et al. Water-soluble contrast media in radiography of small bowel obstruction. Acta Radiol 1988;29(1):53-6. 